\title{
Comment distinguer entre conte et légende : critères internes, critères externes
}

\author{
Michèle Simonsen \\ Københavns Universitet, Copenhague \\ simonsen.michele@gmail.com
}

\section{RÉSUMÉ}

Pour les folkloristes classiques, le principal critère de distinction entre conte et légende est l'opposition entre fiction et croyance. Un conte est tenu pour fictif par le conteur comme par son auditoire; alors que les événements surprenants rapportés dans une légende sont tenus pour vrais (par l'informateur, pas par le chercheur! Mais comment pouvons-nous savoir si le "peuple " croyait à ses légendes?

Les folkloristes moderne ont une conception plus nuancée de la légende, et voient dans celle-ci un énoncé à propos duquel la question de la véracité est pertinente. Alors que la narration d'un conte suscite des réactions de plaisir et des appréciations de type esthétique, la légende suscite des discussions sur sa plausibilité les causes des événements rapportés et la leçon morale à en tirer. L'attention au contexte de la performance est donc essentielle pour distinguer entre conte et légende. Mais lorsque nous cherchons à classifier des documents d'archive collectés il y a plus de cent ans, et profondément remaniés pour la publication, nous n'avons pas de renseignement contextuel à notre disposition. Nous devons nous en tenir aux critères internes. Les légendes disposent de plusieurs procédés rhétoriques pour affirmer ou feindre d'affirmer la véracité des événements qu'elles rapportent.

Toutefois certains récits maintiennent un statut ambigu entre conte et légende. Tel récit facétieux et fictif aux yeux de l'informateur peut être pris pour un récit de croyance par le collecteur. Un récit peut même avoir un statut ambigu pour la communauté. Un conteur doué est parfois plus intéressé par la valeur esthétique d'un récit de croyance que par sa valeur cognitive. On peut peut-être avancer que plus un récit de croyance est esthétiquement élaboré, moins on est en droit de conclure sur la croyance qu'il est censé exprimer.

MOTS-CLE

Conte; légende; folkloristes; critères; croyance 
ABstract

For classical folklorists, the main criterion for distinguishing between tales and legends is the opposition between fiction and belief. A tale is known to be fiction by the story-teller and his audience; whereas the astonishing events reported in a legend are believed to be true (by the folk, but not by the scholar!). But how do we know whether the "folk" believed in their legends?

Modern folklorists have a more nuanced view of legends, and claim that unlike tales, legends are narratives about which the question of truth or untruth is relevant. The telling of a tale causes listeners to express their pleasure and aesthetic appreciation of the story, whereas the telling of a legend leads to discussions about its plausibility, the events it reports and the moral lessons to be drawn from it. Thus, listeners' observations and their scrupulous attention to the performance of stories based on belief are therefore of paramount importance when making the distinction between tales and legends.

However, when we have to classify archive material that was collected some Ioo years ago and that has been altered for publication, such contextual information is missing. We therefore have to resort to internal evidence. Legends use a number of rhetorical tools at to assert the truth of the events they report.

Even so, some stories remain on the border between tale and legend and have an ambiguous status. A story that is regarded is fictitious by an informant may have been taken for a belief-story by the collector. Moreover, a story can sometimes be a border-line story for the community itself. Gifted story-tellers may be more interested in the aesthetic value of a belief story than in its cognitive value. It can be asserted that the more elaborate a legend narrative is, the less we can deduce about the belief it is supposed to express.

\section{KEYWORDS}

Tale; legend; folklorists; criteria; belief

\section{RESUM}

Per als folkloristes clàssics, el criteri principal per distingir històries i llegendes és l'oposició entre la ficció i la creença. Un conte és conegut per la narrativa i el públic, mentre que es creu que els esdeveniments sorprenents descrits en una llegenda són veritables (per part de la gent, però no per l'erudit!). Però, com sabem si la gent creia en les seves llegendes?

Els folkloristes moderns tenen una visió més matisada de les llegendes i afirmen que, a diferència dels contes, les llegendes són narratives sobre les quals la qüestió de la veritat o la falsedat és rellevant. La narració d'un conte genera comentaris de plaer i d'apreciació estètica, mentre que la narració d'una llegenda porta a debats sobre la seva versemblança, l'explicació dels fets reportats i les lliçons morals que s'extrauen. Per tant, l'observació dels participants i l'atenció escrupolosa a l'exercici de les històries de creences són fonamentals per a la distinció entre contes i llegendes.

Però, quan hem de classificar el material d'arxiu recopilat fa uns Ioo anys $i$ alterat per publicar-lo, aquesta informació contextual ens falta. Hem de recórrer a proves internes. Les llegendes tenen a la seva disposició diverses eines retòriques per afirmar la veritat dels esdeveniments sobre els quals informen.

Tot $i$ així, algunes històries es mantenen en la frontera entre el conte i la llegenda $i$ tenen un estatus ambigu. Un relat fictici a ulls de l'informant pot haver estat pres per una història de creença per part del col-lector. A més, una història pot ser, de vegades, un relat de frontera per a la mateixa comunitat. Els narradors dotats poden estar més interessats en el valor estètic d'una història de creença que en el seu valor cognitiu. Es pot afirmar que, com més narrativa sigui la llegenda elaborada, menys podem deduir sobre la creença que se suposa que expressa.

\section{PARAules Clau}

Conte; llegenda; folkloristes; criteris; creença

REBUT: 3I/O3/20I9 | ACCEPTAT: IO/O4/20I9 
$\mathrm{T}$

Tout système de classification est arbitraire, créé par des êtres humains, selon des critères de discrimination plus ou moins subjectifs, qui varient selon les individus et les groupes. Ces catégories ne reflètent donc pas directement la réalité, mais seulement la manière dont un groupe cherche à établir un ordre dans cette réalité. Les catégories ainsi établies reflètent donc les centres d'intérêt et les valeurs de ceux qui les établissent et qui sélectionnent les critères de distinction parmi des dizaines d'autres critères possibles. Ainsi, pour les folkloristes, le couple fiction vs croyance est suffisamment important pour susciter l'opposition entre « conte » et « légende».

De plus, les études folkloriques sont par définition le regard d'un chercheur sur les pratiques d'un groupe. Or les critères distinctifs, et donc les classifications du chercheur, ne sont pas forcément ceux du groupe étudié. Pour la question qui nous concerne aujourd'hui, par exemple, le terme de "légende " au sens ou les folkloristes l'entendent, est en français un terme savant qui, au dix-neuvième siècle encore, n'était jamais utilisé par les usagers. Et pour cause. Pour les chercheurs, une légende est un énoncé de faits irréels mais tenus pour vrais par les locuteurs. Or un même individu ou groupe ne peut pas tenir un énoncé à la fois pour vrai et pour non-vrai. Seuls les autres peuvent croire à des légendes. Par définition, nous ne pouvons pas considérer nos propres légendes - et nous en avons tous - comme des « légendes ».

Par ailleurs, les termes utilisés par les folkloristes ne se recoupent pas toujours d'une langue à l'autre, ni d'une tradition scientifique à l'autre. Or une légende a deux versants: l'expression (le texte, oral ou écrit) et le contenu (la croyance supposée). Dans les traditions nordiques, allemande et anglo-saxonne, les termes de « sage » (allemand), « sagn » (danois) et « legend » (anglais) sont bien plus extensifs que le terme « légende » en français, et recouvrent tous les énoncés légendaires, que ceux-ci soient narratifs ou non. C'est donc au contenu de croyance que le terme s'attache. On a donc été obligé de peaufiner la définition et de distinguer progressivement des sous-classes parmi les «sagn » : avant tout celles du dit, du memorat et du fabulat (von Sydow 1948). Le dit est un énoncé légendaire non narratif, le memorat le récit d'un événement étrange dont le narrateur a été personnellement témoin, ${ }^{\mathrm{I}}$ le fabulat un récit légendaire sans témoin. La distinction entre fabulat et memorat est rejetée par certains folkloristes contemporains (Tangherlini I994). Personnellement, j'estime qu'elle est toujours valide, à condition de bien voir qu'il ne s'agit pas d'une opposition absolue, mais de deux pôles opposés sur une ligne continue. En France au contraire, les folkloristes classiques se sont attachés surtout au versant « récit » de la légende, et ont classé sous ce terme des récits élaborés. Ils ont plutôt classé les énoncés non narratifs sous les termes de croyances, superstitions, etc. Par ailleurs, ils ont publié très peu de memorats, retouchant certainement ceux qu'ils ont entendus pour les publier sous forme de fabulats. Ceci a fourni sans aucun doute des récits plus achevés, mais nous empêche d'analyser avec précision leur degré de « croyance».

Il y aussi une différence générale de nature entre genres savants et genres populaires. Les genres savants classiques (genres poétiques comme genres didactiques) sont théorisés. On est conscient de leurs caractéristiques, régies par des

I Plus tard étendu à un récit personnel dont le narrateur connaît le témoin ou quelqu'un qui le connait. Cf. Granberg (I935). 
Arts poétiques explicites, illustrées par des oeuvres canoniques érigées en exemples à suivre. Un poème est un sonnet ou n'est pas un sonnet. Il ne peut jamais être «plus ou moins » sonnet. En revanche, les genres populaires traditionnels ne sont pas pensés théoriquement, ni créés délibérément en fonction d'un modèle idéal. Leur poétique n'est pas définie ni formulée en termes abstraits. Ils s'inscrivent comme ils le peuvent, au coup par coup, dans une poétique traditionnelle qui n'est jamais formulée, mais qui est rabotée et polie au cours des temps par l'attente, l'appréciation des auditoires et la censure préventive des narrateurs. De sorte qu'un récit traditionnel peut n'être qu'une forme plus ou moins approximative de ce que le chercheur appelle conte ou légende. Un récit traditionnel peut en principe simplement tendre vers le conte ou tendre vers la légende. Autrement dit: si nous avons parfois du mal à classer tel récit parmi les contes ou parmi les légendes, ce n'est pas de notre faute, nous autres folkloristes, c'est souvent « la faute à la réalité ».

Par ailleurs, si les classes établies par les chercheurs sont toujours désignées par un nom («conte», « légende»), celles établies par les usagers ne sont pas toujours formulées explicitement. Elles ne peuvent être décelées qu'indirectement.

Nous sommes presque tous venus aux contes et aux légendes par le biais des études littéraires. Or les littéraires ont l'habitude de définir un genre par deux critères : la forme et le contenu. Mais la littérature orale est avant tout une pratique sociale et les anthropologues nous ont appris à ajouter un troisième critère pour définir un genre : celui du statut de la parole mis en jeu. Or, comme l'ont bien montré Linda Dégh et Andreú Vázsanyi, la narration des légendes est un processus interactif. Le narrateur et son auditoire changent constamment de place. C'est donc le processus de la performance qui constitue la légende (Dégh et Vázsonyi I976: 93-I23). En effet, dans la pratique communautaire, les légendes se fondent dans les conversations ordinaires et n'ont de sens que par les commentaires qu'elles suscitent. Peut-être même la plupart des légendes n'ont-elles jamais été racontées du début jusqu'à la fin ni donné lieu à un récit complet. Peut-être qu'elles vivent d'une vie souterraine, comme un réservoir de motifs et de représentations mentales, et une simple allusion dans la conversation suffit à leur efficacité - du moins dans leur milieu.

Je disais plus haut qu'une légende est un énoncé de faits irréels mais tenus pour vrais par les locuteurs. Les folkloristes modernes ont nuancé cette définition et voient plutôt dans la légende un énoncé de faits remarquables, présentés comme réels, et à propos desquels la question de leur véracité est pertinente, à la différence du « conte », récit toujours tenu pour fictif par les conteurs comme par leur entourage.

Mais comment savoir si un récit est tenu pour vrai ou pour fictif par le narrateur et son auditoire? Nous avons à notre disposition deux types de critères : critères externes et critères internes.

Les critères externes sont avant tout le contexte, la situation de contage, qui est d'une importance primordiale pour le repérage des légendes - beaucoup plus que pour les autres genres de la littérature orale. Idéalement, le chercheur devrait connaître:

- l'identité des membres du groupe en présence, le narrateur et son auditoire (sexe, âge, statut social et professionnel, etc.). 
- les circonstances dans lesquelles se fait le récit: saison, heure et lieu, but de la réunion, activités auxquelles elle donne lieu, etc.

- le déclencheur du récit : quel énoncé d’un locuteur précédent a amené le locuteur à narrer ou évoquer cette légende?

- et surtout les réactions, questions et commentaires de l'auditoire.

Le contexte culturel est aussi très important. La " croyance » supposée exprimée dans le récit a-t-elle des répercussions dans la vie sociale de la communauté? C'est souvent le cas pour les récits de sorcellerie ou de loups-garous, qui ont eu parfois des conséquences dramatiques (mise au ban de la communauté, procès et condamnation, etc.).

Tout ceci est très joli, et depuis l'avènement des " théories sur la performance $",{ }^{2}$ tout collecteur digne de ce nom tient à honneur de noter avec précision la formulation exacte des énoncés et leur contexte d'énonciation. Mais pour nous qui cataloguons, nous travaillons en grande partie sur des matériaux d'archive du dix-neuvième siècle, recueillis et transcrits par des collecteurs qui les ont extraits de leur contexte et grandement manipulés. (Rappelons pour mémoire ce topos des préfaces de recueils folkloriques du dix-neuvième siècle: "Je n'ai rien changé aux récits de mes informateurs, absolument rien, sauf le style, bien entendu »!).

Cela veut-il dire que nous sommes incapables de distinguer entre « légende » et « conte » pour les documents d'archives? Non, bien sûr. Car il y a aussi des critères internes permettant de distinguer entre légende et conte.

Tout d'abord, il y a le contenu. Je ne pense pas tellement aux motifs ou aux personnages, qui souvent figurent aussi bien dans les contes que dans les légendes. Ainsi le récit de la nourriture changée en fleurs, qui constitue le conte AT 7I7*, se retrouve dans les légendes sur Elisabeth de Hongrie, Germaine de Pibrac, etc. Et Saint Pierre, Jésus-Christ et la Vierge Marie figurent dans les légendes chrétiennes comme dans les récits facétieux, les contes religieux et même les contes merveilleux. ${ }^{3}$ Mais ces mêmes personnages ont des caractéristiques différentes dans les contes et dans les légendes. Il y a beaucoup de sorcières dans les contes merveilleux et beaucoup de sorcières dans les récits de sorcellerie. Mais ce ne sont pas du tout les mêmes sorcières! Et Saint-Pierre ou la sorcière jouent des rôles actanciels totalement différents dans les deux types de récit.

De plus, la structure d'un conte n'est pas celle d'une légende. D'une façon générale, un conte finit presque toujours bien, une légende finit souvent mal. Un conte appartient au mode optatif : il a pour fonction d'imaginer comment serait le monde si nous suivions toutes nos pulsions et si tous nos souhaits se réalisaient. Une légende appartient au mode didactique : elle aide l'auditeur à s'orienter par rapport à l'histoire, la géographie locales et les valeurs morales de sa communauté.

Et il y a surtout des procédés d'énonciation propres à la légende. Les légendes se servent d'une rhétorique du vrai, non pas pour affirmer une vérité, mais pour la tester (cf. Goldstein I99I). Elles sont dites d'une certaine manière pour convaincre l'auditoire que le récit est « véridique », ou du moins que la question de sa véracité est pertinente. Du moins en ce qui concerne les memorats.

Considérons par exemple le récit suivant:

2 Cf. Richard Bauman et les nombreux travaux qu'il a inspirés.

3 Cf. les contes ATU 7Io L'enfant de Marie, et le conte français T 3І2B Le Diable et les deux petites filles. 
Ma tante Anne était servante chez Hans Christensen à Rettestrup, dans la paroisse de Vejlø. Hans était un loup-garou, et on en a eu la preuve un jour où lui et sa femme avaient harnaché la carriole pour aller à Noestved. En arrivant au carrefour près de la chaumière de Ole Hansen de Rettestrup, Hans dit à sa femme: "Prends les rênes, et si tu vois arriver une bête, frappe-la de ton tablier à carreaux bleus ». Hans s'est éloigné un moment, et peu de temps après est arrivé un gros chien qui s'est acharné sur sa femme. Elle s'est défendue courageusement, de sorte que le chien n'a pas pu sauter sur ses genoux (sic). Peu de temps après son mari est revenu comme si de rien n'était, a repris place dans la carriole et ils sont rentrés chez eux. Mais le lendemain, alors qu'ils étaient attablés à manger du chou, la femme a remarqué que son mari avait des lambeaux de son tablier entre les dents. Elle s'est exclamée: «Grand Dieu! Mais tu es un loup-garou! » Et Hans a été aussitôt guéri. P. Nielsen, Maître d'école, Skuderløse (Kristensen I928, II: I53).

Il donne des indications précises sur les noms propres des personnes impliquées, des indications précises de lieu et de temps. Le narrateur affirme la véracité des événements racontés en nommant la personne qui en a été témoin, précise le degré de proximité entre le narrateur et ce témoin. Ce sont là les principaux procédés rhétoriques qui distinguent un memorat d'un fabulat. Par conséquent, si l'on veut identifier la croyance derrière le récit (ce qui pour nous folkloristes définit la légende), il vaut mieux avoir affaire à un memorat qu'à un fabulat.

Mais distinguer une légende fabulat d'un conte est beaucoup plus épineux, parce que dans les fabulats nous n'avons aucun de ces procédés rhétoriques à notre disposition. Un fabulat est aussi dénué de localisation qu'un conte.

Il faut reconnaître que, malgré la précision de nos outils d'analyse, il reste des cas-limite entre contes et légendes; des récits dont le statut reste ambigu.

Ce peut être le cas pour le groupe lui-même. Avec les conteurs doués, la fonction esthétique peut l'emporter de beaucoup sur la fonction cognitive. Considérons le récit suivant:

Mon père m'a raconté qu'une fois, il y avait deux femmes qui s'étaient déshabillées et qui s'étaient mises à ramper toutes nues à travers le placenta d'une jument qui avait été étendu entre quatre piquets. Elles étaient enceintes, et elles étaient nues, de sorte que leurs enfants à naître deviendraient des loups-garous. Quand ceux-ci prenaient leur forme animale, ils attaquaient la première personne rencontrée sur leur chemin, quelle qu'elle soit, et la mettaient en pièces. Un valet de ferme de la contrée était allé dans la lande cueillir de la bruyère, avec une servante, qui était sa bonne amie. Une fois arrivés dans la lande, le gars descendit de la carriole et dit à son amie: «Si un loup arrive, je t'en supplie, ne l'attaque pas avec la fourche!» — «Mais alors, avec quoi je me défendrai?» — « Retire ton tablier et frappe le avec ». A peine était-il parti qu'un loup surgit et s'attaqua à la jeune fille. Mais elle le frappa de son tablier, et le loup ne disparut qu'après l'avoir mis en pièces. Peu après le valet revint. "Comme tu as été long! Un loup m'a attaquée et a essayé de me tuer! » Le valet se mit à rire. Alors la servante vit qu'il avait des fils blancs et bleus entre les dents. «C'est donc toi qui m'as attaquée! Tu as encore des fils entre les dents! » 
Et de ce jour là, le valet cessa d'être loup-garou, car son secret avait été dévoilé ». Jens Kristensen, Ersted (Kristensen I980, II: 23I-32).

Ceci est un récit très élégant, du moins en danois. Notez l'alternance entre dialogue et récit direct à la zème personne, entre description et commentaire. Mise à part la formule initiale « Mon père m'a raconté que... » ce récit a la forme d'un fabulat, et ne présente aucun des procédés rhétoriques des memorats pour augmenter la crédibilité. Le narrateur ne semble guère soucieux de convaincre son auditoire de la «vérité » de son récit. Le « mon père m'a dit » initial n'est sans doute qu'un jeu avec les procédés rhétoriques des memorats. Ce récit porte la marque d'une performance longuement peaufinée, et je suppose que, dans son contexte social initial, il a suscité des réactions de plaisir et d'appréciation esthétique plutôt que des discussions sur son contenu; il pourrait même avoir amené un autre conteur doué à enchaîner sur une légende semblable.

En effet les légendes ont un statut flottant sur la ligne qui va de l'acte de parole assertif de la conversation jusqu'à l'acte de parole expressif de la performance artistique. Alors que les contes, contes merveilleux en particulier, sont mis en scène par des performances conscientes d'elles-mêmes de la part de conteurs renommés, performances marquées par des traits remarquables : formules d'ouverture et de clôture, mimique, gestuelle et diction spéciales, etc. La performance des contes donne lieu à des commentaires, des jugements de valeur de nature esthétique ( C'est une belle histoire », « son père aussi était un bon conteur », « tu l'a mieux contée l'autre jour », etc.). Les légendes, au contraire, sont le plus souvent des actes de parole phatiques, des actes de communication, amenés par l'acte de parole précédent et suscitant un autre acte de parole (approbation, question, protestation, etc.). Toutefois, avec les conteurs doués, la fonction esthétique peut l'emporter de beaucoup sur la fonction cognitive. Je serais donc tentée de dire que plus un énoncé légendaire est élaboré, moins il est « vrai », moins on peut inférer le degré de « croyance » qu'il y a derrière.

D'autres phénomènes peuvent aussi compliquer la tâche du classificateur.

Il peut y avoir malentendu, volontaire ou non, entre le collecteur et l'informateur. Le collecteur a peut-être noté comme récit sérieux, donc répertorié comme une "superstition » ou une " croyance », ce qui n'était qu'une facétie de la part de l'informateur. Je pense notamment aux récits de pierre-qui-vire (pierre qui se retourne). Lorsque un informateur du dix-neuvième siècle a dit à un collecteur que la pierre qui est à l'entrée du village se retourne chaque fois qu'elle sent l'odeur de pain en train de cuire, est-ce l'expression de la crédulité des paysans qui ne s'étonnent pas de voir une pierre s'animer? Ou bien est-ce plutôt une manière humoristique d'exprimer le fait que dans ces milieux-là, du pain frais, on n'en mangeait pas souvent? Dans la même veine, je ne peux résister à mentionner un exemple danois qui me ravit. Dans le fichier des Archives de Folklore du Danemark, il y a dans la section « croyances populaires » une carte portant cette simple donnée: «Efter børnenes tro har paven ingen bagdel! ( «Les enfants croient que le pape n'a pas de derrière! »). Cette carte a été rédigée par un archiviste, à qui nous devons sans doute le point d'exclamation, qui semble exprimer une prise de distance par rapport à l'absurdité de la croyance rapportée. Mais s'agit-il vraiment d'une « croyance » ? Si l'on consulte la lettre par laquelle l'informateur inconnu a envoyé cette information aux archives en I948, on voit que l'informateur, un enfant de six ans, a dit textuellement au collecteur : " Tu sais ce que disent les 
autres enfants? Ils disent que le pape n'a pas de derrière ». Il pourrait s'agir tout aussi bien d'une plaisanterie osée, que le petit informateur prend plaisir á répéter tout en en laissant la responsabilité aux " autres enfants ». Ou peut-être se demande -t-il s'il doit prendre au sérieux ce qui n'est qu'une plaisanterie délibérée de la part des plus grands? Ceci me rappelle une des facéties de mon enfance. A la vue d'un prêtre en soutane, les vilains garnements que nous étions se mettaient à crier à tue-tête: «Bonjour madame! » Cette plaisanterie était peut-être d'un goût douteux, mais elle n'exprimait certainement pas la croyance que les prêtres sont des femmes!

Certaines légendes migratoires présentent le phénomène inverse. Un groupe local assigne à un personnage local ou un lieu précis des événements tenus pour véridiques, mais qui circulent un peu partout dans l'espace et le temps, attribués à des personnages et lieux divers, souvent sous forme facétieuse. J'en prendrai pour exemple la célèbre anecdote de «Marie-Antoinette et la brioche ».

Cette anecdote est connue de tous les écoliers de France, où sa véracité n'est jamais mise en cause. Dans ce cas, nous avons affaire à une légende. Mais comme Christine Shojaei Kawan et Véronique Campion-Vincent l'ont montré dans un magnifique article (2002), cette anecdote a été attribuée longtemps avant la Révolution française à de nombreux seigneurs sans pitié pour leurs sujets, pour stigmatiser leur cruauté («Qu'ils mangent de l'herbe! » ou «Qu'ils mangent de la merde! »). Par ailleurs la forme «Qu'ils mangent de la brioche! » a aussi été racontée à propos de seigneurs anonymes, pour se moquer de leur ignorance des réalités sociales. Dans ce cas, la volonté de rire des puissants l'emporte sur l'indignation devant leur dureté, et nous avons affaire à une fiction facétieuse. L'anecdote est d'ailleurs répertoriée comme conte dans la catégorie « Anecdotes and Jokes » ATU I446 Let Them Eat Cake!

Enfin, il peut y avoir des cas-limite entre conte et légende pour le groupe lui-même. Il y a même un genre traditionnel qui a cette ambiguïté comme trait constitutif: le Tall Tale, genre très apprécié en Amérique du Nord, narrant les exploits hyperboliques de héros populaires tels que Paul Bunyan (Etats-Unis) et Big Joe Mufferaw (Canada) (Brown I989). A l'inverse des contes de mensonge hyperboliques à la Münchausen (cf. Raspe I895), affichant ostensiblement leur fictivité, le Tall Tale vise à laisser l'auditeur dans son incertitude: est-ce une histoire vraie, ou une fiction? Le conteur est-il sérieux, ou bien se moque-t-il de nous? Tout le talent du conteur consiste à laisser l'auditoire dans l'incertitude jusqu'au bout.

En conclusion: pour un catalogueur, les cas ambigus entre contes et légendes sont très ennuyeux. Mais pour un amoureux des récits populaires, ils sont le signe de la vivacité d'une tradition qui se joue des catégories étanches établies par les chercheurs. 


\section{Références bibliographiques}

Aarne, Antti ; Stith Thompson (I96i): The Types of the Folktale. A Classification and Bibliography. Helsinki: Suomalainen Tiedeakatemia.

Brown, Carolyn (I989): The Tall Tale in American Folklore and Literature. Knoxville: University of Tennessee Press

CAMPION-Vincent, Veronique et Christine SHOJAei KaWAN (2002) : « Marie-Antoinette et son célèbre dire: deux scénographies et deux siècles de désordres, trois niveaux de communication et trois modes accusatoires ». Annales historiques de la Révolution française no. 327 (javier-mars): 29-56.

DÉGH, Linda ; Andreú VAszonyi (I976) : «Legend and belief ». Dans Dan BeNAmos (ed.): Folklore Genres. Austin \& London : University of Texas Press, p. 93-I23.

Delarue, Paul (I976) : Le Conte populaire français, vol. I. Paris : Maisonneuve et Larose.

Goldstein, Diane (I99I) : «Perspectives on Newfoundland Belief Traditions : Narrative Clues to Concept of Evidence » . Dans Gerald Thomas et J. D. A. Widdowson (ed.) : Studies in Newfoundland Folklore: Community and process. St. John's: Newfoundland, p. 27-40.

Granberg, Gunnar (I935) : « Memorat und Sage. Einige Methodischegesichtepunkte ». Dans Saga och sed. Gustav Adolfs Akademiens ârsbok. Uppsala: A.-B. Lundequistska Bokhandeln: I2O-I27.

KRISTENSEN, Ewald Tang (I892-I90i) (I980) : Danske Sagn som de har lydt $i$ folkemund. Udelukkende efter utrykte kilder. 8 vols. Traduit par M. Simonsen. Copenhagen: Arnold Busk.

- (I928) : Danske Sagn som de har lydt i folkemund. Udelukkende efter utrykte kilde: ny roekke, edited by Johannes Evald Tang Kristensen. Traduit par M. Simonsen. Copenhagen: Arhus folkeblads trykkeri.

RASPE, Rudolph Erich [I785] (I895): The surprising Adventures of Baron Münchausen. London: Lawrence and Bullen.

Sydow, Carl-Vilhelm von (I948) : Selected papers on Folklore. Copenhagen : Rosenkilde \& Bagger.

TANGHERLINI, Timothy (I994) : Interpreting Legend. Danish Storytellers and their Repertoires. New-York: Garland.

UTHER, Hans-Jörg (2004): The Types of International Folktales. A Classification and Bibliography. Helsinki: Suomalainen Tiedeakatemia. 